\title{
ON A CALCULUS OF OPERATORS IN REFLEXIVE VECTOR SPACES*
}

\author{
BY \\ EDGAR R. LORCH

\section{INTRODUCTION}

Linear transformation theory in general vector spaces is not nearly as extensive as it is for that special space, Hilbert space. In Hilbert space large and important classes of transformations, the self-adjoint and unitary transformations, may be studied exhaustively because these transformations are susceptible of a spectral resolution. In turn, the spectral theory leads to an elegant calculus of these transformations or operators which asserts the existence of a ring homomorphism between a class of functions and a class of permutable operators centered about a given operator. These developments are possible because Hilbert space-is self-adjoint.

Other vector spaces have not to the present yielded such rich results. The operators of no important class have been found to be completely resolvable. Indeed, a theory of projections which is the first step toward a spectral development has not to our knowledge been given, although the matter has received attention before this. In his investigations in the problem of complementary manifolds in the spaces $L_{p}$ and $l_{p}, \mathrm{~F}$. J. Murray introduces the notion of projection at an early stage. $\dagger$ These investigations establish the existence of manifolds which do not generate projections. We show that we need never consider such manifolds if they are avoided at the outset, for the operations we perform do not lead to them.

This paper treats first the subject of projections in spaces of a rather general type. The reflexive property (see definition below) $\ddagger$ is assumed in order to insure the existence of a limit for monotone sequences of projections. This leads to the establishment of the existence of least upper and greatest lower bounds of sets of permutable projections. Subsequently, a calculus is developed for operators which are defined by means of a resolution of the identity. This calculus possesses properties as extensive as those found in

* Presented to the Society, February 26, 1938; received by the editors December 31, 1937.

$\dagger$ F. J. Murray, On complementary manifolds and projections in the spaces $L_{p}$ and $l_{p}$, these Transactions, vol. 41 (1937), pp. 138-152.

¥ We use the word "reflexive" in preference to "regular" which was introduced by H. Hahn, Über lineare Gleichungssysteme in linearen Railmen, Journal für die reine und angewandte Mathematik, vol. 157, pp. 214-229. 
Hilbert space.* The problem of characterizing in simple fashion operators which may be resolved is still open. It may be of interest to note that preliminary attempts in that direction incline one to an optimistic outlook.

\section{The ALgebra of PROJections}

1. The space. We operate in a normed linear vector space $\mathfrak{B}$ whose elements will be designated by $f, g, h, \cdots$. Addition of elements and multiplication by complex numbers (denoted by $\rho, \sigma, \tau, \cdots$ ) is permitted subject to the customary restrictions. The norm of $f,\|f\|$, is a real-valued function which satisfies the conditions $\|f\| \geqq 0,\|f\|=0$ implies $f=0,\|\rho f\|=|\rho|\|f\|$, and $\|f+g\| \leqq\|f\|+\|g\|$. If we write distance $(f, g)=\|f-g\|$, the norm metrizes $\mathfrak{B}$. If the sequence $\left\{f_{n}\right\}$ converges to $f$ in this metric, we often write $f_{n} \rightarrow f$ for $\left\|f-f_{n}\right\| \rightarrow 0$. The space $B$ is assumed to be complete in this metric. $\dagger$

An operation $O$ is a function whose domain is the space $\mathscr{B}$ and whose range is a subset of a space of the same type $\mathfrak{B}_{1} . O$ is said to be distributive if $O(\rho f+\sigma g)=\rho O f+\sigma O g$. If $O$ is distributive and continuous at every point of $\mathscr{B}$, then $O$ is said to be linear. It is known that if $O$ is linear (and only then if $O$ is distributive), there exists a constant $K \geqq 0$ independent of $f \varepsilon \mathfrak{B}$, such that $\|O f\| \leqq K\|f\|$. The least such constant $K$ is called the bound of $O$. The operation $O$ is said to be closed if $f_{n} \rightarrow f, O f_{n} \rightarrow g$ implies $O f=g$. A closed distributive operation is linear.

An operation whose range is contained in the initial space $B$ is called an operator or transformation. Operators are denoted by $A, B, P, Q, \cdots$. Two special operators and their defining equations are $0, I$, with $0 f=0, I f=f$. The bounds of $A, P, \cdots$ are written $|A|,|P|, \cdots$.

An operation whose range is a set of complex numbers is called a functional. Functionals will be denoted by $F, G, \cdots$. The totality of linear functionals defined on $\mathfrak{B}$ is a linear set. If we write $\|F\|$ for the bound of $F$, the set of functionals is a complete space $(\mathfrak{B})$ of the same type as $\mathscr{B}$. The space $(\mathfrak{B})$ is said to be the space adjoint to $\mathfrak{B}$. The space $((\mathfrak{B}))$ may now be introduced in evident fashion.

If $\mathrm{A}$ is a linear operator, and $F$ is a linear functional, both defined on $\mathfrak{B}$, then $F(A)$ is a linear functional. Indeed, $F(A[f+g])=F(A f+A g)=F(A f)$ $+F(A g)$. Also

* For recent developments in the theory of the operational calculus in Hilbert space cf. J. von Neumann, Über Funktionen von Funktionaloperatoren, Annals of Mathematics, (2), vol. 32 (1931), pp. 191-226; M. H. Stone, Linear Transformations in Hilbert Space and their Applications to Analysis, American Mathematical Society Colloquium Publications, vol. 15, New York, 1932, chap. 6.

† These spaces form the subject of Banach's treatise Theorie des Opérations Linéaires. We shall make free and constant use of results there discussed. That these results are for the most part valid in complex spaces has been pointed out recently by various authors. 


$$
|F(A f)| \leqq\|F\| \cdot\|A f\| \leqq\|F\| \cdot|A| \cdot\|f\| .
$$

The correspondence $F$ to $F(A)$ defined over $(\mathfrak{B})$ is linear; for it is distributive and we have just seen that it is bounded. This linear correspondence is written in the operator form, $G=\bar{A}(F)$. It is easy to prove that $|\bar{A}|=|A|$, $(\overline{A+B})=\bar{A}+\bar{B},(\overline{A \cdot B})=\bar{B} \cdot \bar{A}$. The operator $\bar{A}$ is called the adjoint of $A$.

Consider now the spaces $\mathfrak{B},(\mathfrak{B}),((\mathfrak{B}))$. If $f_{\varepsilon} \mathfrak{B}$ is fixed and $F \varepsilon(\mathfrak{B})$ is variable, $\Phi(F)=F(f)$ is an element of $((\mathfrak{B}))$. For $\Phi$ is distributive and $|\Phi(F)|=|F(f)|$ $\leqq\|f\| \cdot\|F\|$; hence $\Phi$ is linear. From this it also follows that $\|\Phi\| \leqq\|f\|$. Since a $G$ \& (B) exists such that $G f=\|f\|,\|G\|=1$, we have $|\Phi(G)|=|G(f)|=\|f\|$ $\leqq\|\Phi\| \cdot\|G\|=\|\Phi\|$; we must conclude that $\|\Phi\|=\|f\|$. The correspondence $f$ to $\Phi$ is a linear isometric map of $\mathscr{B}$ on a subset of $((\mathscr{B}))$. A space is said to be reflexive if the range of this correspondence is $((\mathscr{B}))$ in its entirety. If $\mathscr{B}$ is reflexive, we write, for short, $((\mathfrak{B}))=\mathfrak{B}$.

We assume that the space $\mathfrak{B}$ is reflexive.

2. Manifolds. A set $M$ of elements is said to be linear if, when $f, g \in M$, $f+g \varepsilon M, \rho f \varepsilon M, \rho$ a complex number. If $M$ is linear and closed, it is called a closed linear manifold, or for short, a manifold. Manifolds will be designated by the letters $\mathfrak{M}, \mathfrak{R}, \cdots$. Let $\left\{\mathfrak{M}_{\alpha}\right\}$ be any set of closed linear manifolds. Then there exists a smallest manifold $\mathfrak{M}$ containing each $\mathfrak{M}_{\alpha}$; we denote this by writing $\mathfrak{M}=\sum_{\alpha}^{*} \mathfrak{M}_{\alpha}$ (or, in case the index $\alpha$ ranges over two elements only, $\mathfrak{M}=\mathfrak{M}_{1}+\mathfrak{M}_{2}$ ). The largest manifold $\mathfrak{N}$ contained in each $\mathfrak{M}_{\alpha}$ is precisely the set intersection of the $\mathfrak{M}_{\alpha}, \mathfrak{N}=\prod_{\alpha} \mathfrak{M}_{\alpha}$ (or, as above, $\mathfrak{N}=\mathfrak{M}_{1} \cdot \mathfrak{M}_{2}$ ).

The elements $f_{\varepsilon} \mathfrak{B}, F_{\varepsilon}(\mathfrak{B})$, are said to be orthogonal to each other if $F(f)=0$. If $\mathfrak{M} \subset \mathfrak{B}$ is any manifold, the set of all elements $F \varepsilon(\mathfrak{B})$ orthogonal to each element of $\mathfrak{M}$ is a closed linear manifold $(\mathfrak{R})$. Such a manifold $(\mathfrak{R})$ is called the orthogonal complement of $\mathfrak{M}$ and is denoted by $\mathfrak{M}^{\perp}$. If each element of $\mathfrak{M}$ is orthogonal to each element of $(\mathfrak{R})$, we write $\mathfrak{M} \perp(\mathfrak{R})$. If $(\mathfrak{R})$ is any manifold in $(\mathfrak{B})$, by the orthogonal complement $\mathfrak{N}$ of $(\mathfrak{M})$, we mean the totality of elements in $\mathfrak{B}$ orthogonal to $(\mathfrak{M})$. We note that for $\mathfrak{N} \subset \mathfrak{B}, \mathfrak{N}^{\perp \perp}=\mathfrak{N}$; here $\mathfrak{N}^{\perp \perp}$ means $\Re^{\perp}$ where $\Re=\mathfrak{N}^{\perp}$. For clearly $\mathfrak{N}^{\perp \perp} \supset \mathfrak{l}$. Let us assume that $f \varepsilon \mathfrak{N}^{\perp \perp}, f \in \mathfrak{N}$. Then there exists an $F \varepsilon(\mathfrak{B})$ such that $F f=1, F \perp \mathfrak{R}$. Thus $F \varepsilon \mathfrak{R}^{\perp}$; hence $F f=0$. We conclude that $\mathfrak{N}^{\perp \perp}=\mathfrak{R}$. If $(\mathfrak{R}) \subset(\mathfrak{B})$, then clearly $(\mathfrak{R})^{\perp \perp} \supset(\mathfrak{R})$. To establish the equality of these manifolds, we rely on the reflexive character of the space. Assume $F \varepsilon(\mathfrak{R})^{\perp \perp}, F \in(\mathfrak{R})$. Then there exists an $f \varepsilon \mathfrak{B}$ such that $f \perp(\mathfrak{R}), F(f)=1$; but this means that $f_{\varepsilon}(\mathfrak{R})^{\perp}$ and $F f=0 \neq 1$. Thus $(\mathfrak{R})^{\perp \perp}=(\mathfrak{N})$.

We terminate this section with a definition and theorem centering about the operation $\dot{+}$. Let $\mathfrak{M}$ and $\mathfrak{N}$ be two closed linear manifolds which have the property that there exists a constant $k>0$ such that for every $f_{\varepsilon} \mathfrak{M}$ and $g \varepsilon \mathfrak{R}$, 
$\|f+g\| \geqq k\|f\|$. Then there exists a constant $k^{\prime}>0$ such that $\|f+g\| \geqq k^{\prime}\|g\|$. In fact, the inequality $\|f+g\| \geqq\|g\|-\|f\|$ together with the assumed inequality yields $(1+k)\|f+g\| \geqq k\|g\|$ which is what we desire with $k^{\prime}$ equal to $k /(1+k)$. The symmetry in the roles of $\mathfrak{M}$ and $\mathfrak{N}$ allows us to frame the following definition:

Definition 1. Two closed linear manifolds $\mathfrak{M}$ and $\mathfrak{R}$ will be said to be disjoint if there exists a constant $k>0$ such that for every $f_{\varepsilon} \mathfrak{M}$ and for every $g \& \mathfrak{R}$, $\|f+g\| \geqq k\|f\|$.

THEOREM 2.1. Two closed linear manifolds $\mathfrak{M}$ and $\mathfrak{N}$ are disjoint if and only if they satisfy the following conditions:

(1) The manifolds have only the element zero in common.

(2) The set of all elements of the form $f+g, f \in \mathfrak{M}, g \in \mathfrak{R}$, is a closed linear manifold.

We assume $\mathfrak{M}$ and $\mathfrak{N}$ disjoint. Let $f \varepsilon \mathfrak{M} \cdot \mathfrak{R}$. Then $-f \varepsilon \mathfrak{R}$, and by the foregoing definition $0=\|f-f\| \geqq k\|f\|$ with $k>0$. Hence $\|f\|=0, f=0$. This establishes (1). In proving (2), we note that the set of elements of the form $f+g$ is linear. Suppose $h_{n}=f_{n}+g_{n},(n=1,2, \cdots)$, and that $h_{n} \rightarrow h$. We have $\left\|h_{n}-h_{m}\right\| \geqq k\left\|f_{n}-f_{m}\right\|$; since $\left\|h_{n}-h_{m}\right\| \rightarrow 0,\left\|f_{n}-f_{m}\right\| \rightarrow 0$. The sequence $\left\{f_{n}\right\}$ is convergent to an element $f$ which is in $\mathfrak{M}$ since $\mathfrak{M}$ is closed. This implies $g_{n} \rightarrow g \varepsilon \Re$. Thus $h=f+g$ and (2) is established.

We now assume conditions (1) and (2). The manifold of all elements of the form $f+g$ is a complete linear space $\mathbb{E}$ of the same type as $\mathscr{B}$. (1) implies directly that all elements in $\&$ can be expressêd in the form $f+g$ in only one way. The operator $A$ which carries $f+g$ into $f, A(f+g)=f$, is distributive. Furthermore the conditions $h_{n}=f_{n}+g_{n},(n=1,2, \cdots), h_{n} \rightarrow h, f_{n} \rightarrow f$ in $\mathfrak{M}$ imply $g_{n} \rightarrow g$ in $\mathfrak{R}$, where $h=f+g$. Thus the operator $A$ is closed. It is therefore linear. Choose $k>0$ so that $\|A h\| \leqq\|h\| / k$. Then $k\|A(f+g)\|=k\|f\| \leqq\|f+g\|$. This terminates the proof.

3. Projections. This section is devoted to the development of an elementary theory of projections in $\mathscr{B}$.

Definition 2. A linear operator $P$ is called a projection if $P^{2}=P$.

ThEOREM 2.2. $\dagger$ Let $P$ be any projection in $\mathfrak{B}$; let $\mathfrak{M}$ be the set of elements $f$ for which $P f=f ;$ let $\mathfrak{N}$ be the set of elements $g$ for which $P g=0$. Then $\mathfrak{M}$ and $\mathfrak{N}$ are disjoint closed linear manifolds and $\mathfrak{M} \mathfrak{+} \mathfrak{N}=\mathfrak{B}$. Conversely, if $\mathfrak{M}$ and $\mathfrak{R}$ are disjoint closed linear manifolds for which $\mathfrak{M} \mathfrak{+}=\mathfrak{B}$, there exists a unique projection $P$ which satisfies the equations $P f=f, f \varepsilon \mathfrak{M} ; P g=0, g \varepsilon \mathfrak{N}$. loc. cit.

$\dagger$ The proof of this theorem resembles closely that of Lemma 1.1.1, p. 138, given by Murray 
Let $P$ be a projection. We note that if $f, g \varepsilon \mathfrak{M}, \rho f, f+g \varepsilon \mathfrak{M}$. Furthermore, since $P$ is continuous, $\mathfrak{M}$ is closed; thus $\mathfrak{M}$ is a manifold. Similarly, $\mathfrak{R}$ is a manifold. If $f_{\varepsilon} \mathfrak{B}$, then $f=P f+(f-P f)$. Since $P^{2}=P, P f \varepsilon \mathfrak{M}, f-P f \varepsilon \mathfrak{R}$. Thus $\mathfrak{M} \dot{+} \mathfrak{R}=\mathfrak{B}$. Since $\|P f\| \leqq|P| \cdot\|f\|=|P| \cdot\|P f+(f-P f)\|, \mathfrak{M}$ and $\mathfrak{N}$ are disjoint.

We turn to the converse. Let $\mathfrak{M}$ and $\mathfrak{R}$ be disjoint, $\mathfrak{M} \mathfrak{+} \mathfrak{R}=\mathfrak{B}$. Let $h \varepsilon \mathfrak{B}$, $h=f+g, f \varepsilon \mathfrak{M}, g \varepsilon \mathfrak{R}$. Then the operator $P$ for which $P h=f$ is distributive. As in Theorem 2.1, $P$ is closed, hence linear. Furthermore, $P^{2} h=P(P h)=P f=f$ $=P h$. Thus $P$ has the properties required by the theorem. Any linear operator which is identical with $P$ on $\mathfrak{M}$ and on $\mathfrak{R}$ is identical with $P$ in $\mathfrak{B}$ since $\mathfrak{M} \dot{+} \mathfrak{N}=\mathfrak{B}$.

The manifolds $\mathfrak{M}$ and $\mathfrak{N}$ described above are said to be associated to the projection $P$. We shall sometimes denote the manifolds associated to $P_{1}$ and $P_{2}, \cdots$ by $\mathfrak{M}_{P_{1}}, \mathfrak{N}_{P_{1}}, \cdots$, also by $\mathfrak{M}_{1}, \mathfrak{N}_{1}, \cdots$.

Theorem 2.3. If $P$ is a projection in $\mathfrak{B}$, then $\bar{P}$ is a projection in (B). If $\mathfrak{M}, \mathfrak{R}$ and $(\mathfrak{M}),(\mathfrak{N})$ are the manifolds associated to $P$ and $\bar{P}$, respectively, then $(\mathfrak{M})=\mathfrak{N}^{\perp}$ and $(\mathfrak{N})=\mathfrak{M}^{\perp}$.

The relation $P^{2}=P$ implies $(\overline{P \cdot P})=\bar{P}^{2}=\bar{P}$; thus $\bar{P}$ is a projection. $\bar{P}$ is defined by the equations $\bar{P}(F)=G$ where $G f=F(P f)$. Let $G \varepsilon(\mathfrak{M})$. Then $G=\bar{P}(G)$ and $G f=G(P f)=0$ if $f \varepsilon \mathfrak{R}$. Thus $(\mathfrak{M}) \perp \mathfrak{N}$ or $(\mathfrak{M}) \subset \mathfrak{N}^{\perp}$. Now let $G$ be any element orthogonal to $\mathfrak{R}$. We shall show that $G=\bar{P}(G)$. Let $f$ be arbitrary in $\mathfrak{B}$. Then $G f=G(P f+(f-P f))=G P f+G(f-P f)=G(P f)$ since $f-P f \varepsilon \mathfrak{N}$. Thus $(\mathfrak{M})=\mathfrak{N}^{\perp}$. The proof that $(\mathfrak{N})=\mathfrak{M}^{\perp}$ is similar.

ThEOREM 2.4. If $P_{1}$ and $P_{2}$ are projections and $\mathfrak{M}_{1}, \mathfrak{R}_{1}, \mathfrak{M}_{2}, \mathfrak{N}_{2}$ are their associated manifolds, then

(1) $P_{1} \cdot P_{2}$ is a projection if and only if $P_{1}\left(\mathfrak{M}_{2}\right) \subset \mathfrak{M}_{2} \dot{+} \mathfrak{N}_{1} \mathfrak{N}_{2}$;

(2) $P_{1}+P_{2}$ is a projection if and only if $P_{1} P_{2}=P_{2} P_{1}=0$.

(1) Assume $P_{1} P_{2}$ a projection. Let $f \varepsilon \mathfrak{M}_{2}, P_{1} f=g+h, g \varepsilon \mathfrak{M}_{2}, h \varepsilon \mathfrak{N}_{2}$. Then $P_{1} f=P_{1} P_{2} f=P_{1} P_{2} P_{1} P_{2} f=P_{1} P_{2} P_{1} f=P_{1} g$. Thus $P_{1}(f-g)=0, f-g=f-P_{1} f+h$ $\varepsilon \mathfrak{R}_{1}$, or $h \varepsilon \mathfrak{N}_{1}$. This establishes that $P_{1}\left(\mathfrak{M}_{2}\right) \subset \mathfrak{M}_{2} \dot{+} \mathfrak{N}_{1} \mathfrak{N}_{2}$.

Now assume that $P_{1}\left(\mathfrak{M}_{2}\right) \subset \mathfrak{M}_{2}+\mathfrak{R}_{1} \mathfrak{N}_{2}$. Note that $P_{1} P_{2}$ is distributive and bounded, hence linear. We show that $\left(P_{1} P_{2}\right)^{2}=P_{1} P_{2}$. The equation holds on $\mathfrak{N}_{2}$. It will suffice to establish it on $\mathfrak{M}_{2}$. For $f \varepsilon \mathfrak{M}_{2}, P_{1} f=g+h, g \varepsilon \mathfrak{M}_{2}, h \varepsilon \mathfrak{N}_{1} \mathfrak{N}_{2}, t$ $P_{1} P_{2} P_{1} P_{2} f=P_{1} P_{2} P_{1} f=P_{1} g=P_{1}(g+h)=P_{1} f=P_{1} P_{2} f$. This completes the proof of part (1).

$\dagger$ The manifolds $\mathfrak{M}_{2}$ and $\mathfrak{N}_{2}$ are disjoint. Thus $\mathfrak{M}_{2}$ and $\mathfrak{N}_{1} \mathfrak{N}_{2}$ are disjoint. By Theorem 2.1, any element in $\mathfrak{M}_{2}+\mathfrak{N}_{1} \mathfrak{N}_{2}$ has the form $g+h$ which we describe above. 
(2) We note that $\left(P_{1}+P_{2}\right)^{2}=P_{1}+P_{2}+P_{1} P_{2}+P_{2} P_{1}$. If $P_{1} P_{2}=P_{2} P_{1}=0$, $P_{1}+P_{2}$ is a projection. Assume now that $P_{1}+P_{2}$ is a projection. Then $P_{1} P_{2}+P_{2} P_{1}=0$. We shall see that $P_{2} P_{1}=0$. Note that $P_{2} P_{1}=0$ if $f \varepsilon \Re_{1}$. If $f \varepsilon \mathfrak{M}_{1}, P_{1} P_{2} f+P_{2} P_{1} f=P_{1} P_{2} f+P_{2} f=0$. Let $P_{2} f=g+h, g \varepsilon \mathfrak{M}_{1}, h \varepsilon \mathfrak{N}_{1}$, then $P_{1} P_{2} f+P_{2} f=g+g+h=0,2 g=-h, g=h=0, P_{2} f=P_{2} P_{1} f=0$.

If for the projections $P_{1}$ and $P_{2}, \mathfrak{M}_{1} \supset \mathfrak{M}_{2}, \mathfrak{N}_{1} \subset \mathfrak{N}_{2}$, we write $P_{1}>P_{2}$. If $P_{1}>P_{2}$ and $P_{2}>P_{3}$, then $P_{1}>P_{3}$. If $P_{1}>P_{2}$, then $\bar{P}_{1}>\bar{P}_{2}$. If $P_{1}>P_{2}$, then $P_{1} P_{2}=P_{2} P_{1}=P_{2}$. For any projection $P, I>P>0$.

THEOREM 2.5. If $P_{1}$ and $P_{2}$ are permutable projections, then $P_{1} P_{2}$ and $Q=P_{1}+P_{2}-P_{1} P_{2}$ are projections. The associated manifolds of these projections are related by the equations $\mathfrak{M}_{P_{1} P_{2}}=\mathfrak{M}_{P_{1}} \cdot \mathfrak{M}_{P_{2}}, \mathfrak{N}_{P_{1} P_{2}}=\mathfrak{N}_{P_{1}}+\mathfrak{N}_{P_{2}} ;$ $\mathfrak{M}_{Q}=\mathfrak{M}_{P_{1}}+\mathfrak{M}_{P_{2}}, \mathfrak{N}_{Q}=\mathfrak{N}_{P_{1}} \cdot \mathfrak{N}_{P_{2}}$. If $P_{1}>P_{2}$, then $R=P_{1}-P_{2}$ is a projection and $\mathfrak{M}_{R}=\mathfrak{M}_{P_{1}} \cdot \mathfrak{R}_{P_{2}}, \mathfrak{N}_{R}=\mathfrak{M}_{P_{2}}+\mathfrak{N}_{P_{1}}$.

Direct computation yields $\left(P_{1} P_{2}\right)^{2}=P_{1} P_{2}, Q^{2}=Q$, and if $P_{1}>P_{2},\left(P_{1}-P_{2}\right)^{2}$ $=P_{1}-P_{2}$. Clearly, $\mathfrak{M}_{P_{1} P_{2}} \supset \mathfrak{M}_{P_{1}} \cdot \mathfrak{M}_{P_{2}}$. Now let $P_{1} P_{2} f=f$. Then $f=P_{1} P_{2} f$ $=P_{1} P_{2}^{2} f=P_{2} P_{1} P_{2} f=P_{2} f ;$ thus $f \varepsilon \mathfrak{M}_{2}$. Similarly $f \varepsilon \mathfrak{M}_{1}$. Therefore $\mathfrak{M}_{P_{1} P_{2}}$ $=\mathfrak{M}_{P_{1}} \cdot \mathfrak{M}_{P_{2}}$.

Next, $\mathfrak{N}_{P_{1} P_{2}} \supset \mathfrak{N}_{P_{1}}, \mathfrak{N}_{P_{1} P_{2}} \supset \mathfrak{N}_{P_{2}}$; hence $\mathfrak{N}_{P_{1} P_{2}} \supset \mathfrak{N}_{P_{1}} \dot{+} \mathfrak{N}_{P_{2}}$. Let $P_{1} P_{2} f=0$, $f=g+h, g \varepsilon \mathfrak{M}_{2}, h \varepsilon \mathfrak{R}_{2}$. Then $P_{1} P_{2} f=P_{1} g=0, g \varepsilon \mathfrak{N}_{1}$, and $f=g+h \varepsilon \mathfrak{N}_{1} \dot{+} \mathfrak{N}_{2}$. Hence $\mathfrak{N}_{P_{1} P_{2}}=\mathfrak{N}_{P_{1}}+\mathfrak{N}_{P_{2}}$.

As for $Q$, let $f \varepsilon \mathfrak{M}_{P_{1}}, g \varepsilon \mathfrak{M}_{P_{2}}$, then $Q(f+g)=f+P_{1} g+P_{2} f+g-P_{1} P_{2} f$ $-P_{1} P_{2} g=f+g$ since $P_{1} g+P_{2} f=P_{1} P_{2} g+P_{1} P_{2} f$. Hence $\mathfrak{M}_{Q} \supset \mathfrak{M}_{P_{1}} \dot{+} \mathfrak{M}_{P_{2}}$. If $Q f=f$, write $f=g+h, g \varepsilon \mathfrak{M}_{1}, h \varepsilon \mathfrak{N}_{1}, Q f=g+P_{2} g+P_{2} h-P_{2} P_{1}(g+h)=g+P_{2} g$ $+P_{2} h-P_{2} g=g+h ; P_{2} h=h, h \varepsilon \mathfrak{M}_{2}$, and $f \varepsilon \mathfrak{M}_{1}+\mathfrak{M}_{2}$.

Next, note that $\mathfrak{R}_{Q} \supset \mathfrak{N}_{P_{1}} \cdot \mathfrak{N}_{P_{2}}$. If $f \varepsilon \mathfrak{R}_{Q}$, then $Q f=\left(P_{1}+P_{2}-P_{1} P_{2}\right) f=0$, $P_{1} f=\left(P_{1}-I\right) P_{2} f=P_{1}^{2} f=\left(P_{1}-I\right) P_{1} P_{2} f=0$, and $f \varepsilon \mathfrak{N}_{1}$; likewise $f \varepsilon \mathfrak{N}_{2}$. Hence $\mathfrak{N}_{Q}=\mathfrak{N}_{P_{1}} \cdot \mathfrak{R}_{P_{2}}$.

We consider $R$. Clearly, $\mathfrak{M}_{R} \supset \mathfrak{M}_{P_{1}} \cdot \mathfrak{N}_{P_{2}}$. If $\left(P_{1}-P_{2}\right) f=f, P_{2} f=P_{2}\left(P_{1}-P_{2}\right) f$ $=0, f \varepsilon \mathfrak{N}_{P_{2}}$, and hence $f \varepsilon \mathfrak{M}_{P_{1}}$. Note that $R=0$ on $\mathfrak{M}_{P_{2}}$ and on $\mathfrak{N}_{P_{1}}$. And if $R f=0$, then $P_{1} f=P_{2} f, f=P_{2} f+\left(f-P_{2} f\right)=P_{2} f+\left(f-P_{1} f\right)$; and since $P_{2} f \varepsilon \mathfrak{M}_{P_{2}}$, $f-P_{1} f \varepsilon \mathfrak{N}_{P_{1}}$, we must have $\mathfrak{N}_{R}=\mathfrak{M}_{P_{2}}+\mathfrak{N}_{P_{1}}$.

\section{INFINITE SYSTEMS OF PROJECTIONS}

In the beginning of this chapter, monotone sequences of projections are treated. We determine conditions under which a limit operator exists. Subsequently, the notion of least upper bound and greatest lower bound of sets of permutable projections is examined.

We remark first that if $\mathfrak{M}$ and $\mathfrak{N}$ are disjoint, and if there exists an $f(\neq 0) \varepsilon \mathfrak{B}$ not in $\mathfrak{M} \dot{+} \mathfrak{R}$, then there exists an $F(\neq 0) \varepsilon \mathfrak{M}^{\perp} \cdot \mathfrak{R}^{\perp}$. Thus if $\mathfrak{M}^{\perp}$ 
and $\mathfrak{N}^{\perp}$ are disjoint, $\mathfrak{M} \mathfrak{+} \mathfrak{N}=\mathfrak{B}$. In proof, let $f \mathfrak{M} \mathfrak{+} \mathfrak{N},(f \neq 0)$; then there exists an $F_{\varepsilon}(\mathfrak{B})$ such that $F f=1, F \perp \mathfrak{M} \dot{+}$. Hence we have $F(\neq 0) \varepsilon \mathfrak{M}^{\perp}$, and $F \varepsilon \mathfrak{R}^{\perp}$.

Theorem 3.1. Let $\left\{P_{n}\right\}$ be a sequence of projections for which $P_{n}<P_{n+1}$, $\left|P_{n}\right| \leqq K,(n=1,2, \cdots)$. Let the adjoint of $P_{n}$ be $\bar{P}_{n}$, and let $\mathfrak{M}_{n}, \mathfrak{R}_{n},(\mathfrak{M})_{n}$, $(\mathfrak{N})_{n}$ denote the manifolds associated to $P_{n}$ and $\bar{P}_{n}$. Let $\mathfrak{M}=\sum_{1}^{\infty}{ }^{*} \mathfrak{M}_{\alpha}, \mathfrak{N}=\prod_{1}^{\infty} \mathfrak{N}_{\alpha}$, $(\mathfrak{M})=\sum_{1}^{\infty} *(\mathfrak{M})_{\alpha},(\mathfrak{R})=\prod_{1}^{\infty}(\mathfrak{R})_{\alpha}$. Then

(1) $\mathfrak{M}$ and $\mathfrak{R}$ are disjoint, $(\mathfrak{M})$ and $(\mathfrak{R})$ are disjoint;

(2) $(\mathfrak{M})=\mathfrak{R}^{\perp},(\mathfrak{R})=\mathfrak{M}^{\perp} ; \mathfrak{M}=(\mathfrak{R})^{\perp}, \mathfrak{R}=(\mathfrak{M})^{\perp}$;

(3) $\mathfrak{M} \dot{+} \mathfrak{R}=\mathfrak{B} ;(\mathfrak{M}) \dot{+}(\mathfrak{R})=(\mathfrak{B})$.

(1) Let $f \varepsilon \mathfrak{M}, g \varepsilon \mathfrak{R}$. Then there exist elements $f_{n} \varepsilon \mathfrak{M}_{n}$ such that $f_{n} \rightarrow f$. Thus $\left\|P_{n}\left(f_{n}+g\right)\right\|=\left\|f_{n}\right\| \leqq K\left\|f_{n}+g\right\|$. As the case $\left|P_{n}\right|=0,(n=1,2, \cdots)$, is trivial, we assume $K>0$. Thus $\left\|f_{n}+g\right\| \geqq\left\|f_{n}\right\| / K$; therefore $\|f+g\| \geqq\|f\| / K$ which implies the disjointness of $\mathfrak{M}$ and $\mathfrak{N}$. To show the same for (M) and $(\mathfrak{N})$, we observe that $\left|\bar{P}_{n}\right|=\left|P_{n}\right| \leqq K, \bar{P}_{n}<P_{n+1},(n=1,2, \cdots)$, and apply the result just obtained.

(2) Since $(\mathfrak{R})_{n}=\mathfrak{M}_{n}{ }^{1}$ by Theorem $2.3, \mathfrak{M}_{n} \perp(\mathfrak{R}) \subset(\mathfrak{R})_{n},(n=1,2, \cdots)$. Hence, $(\mathfrak{N}) \perp \sum_{1}^{\infty} * \mathfrak{M}_{\alpha}=\mathfrak{M}$, or $(\mathfrak{R}) \subset \mathfrak{M}^{\perp}$. Now let $F \varepsilon \mathfrak{M}^{\perp}$. Then $F \perp \mathfrak{M}_{n}$; hence $F_{\varepsilon}(\mathfrak{R})_{n},(n=1,2, \cdots), F_{\varepsilon} \prod_{1}^{\infty}(\mathfrak{R})_{\alpha}=(\mathfrak{R})$. Thus $(\mathfrak{R})=\mathfrak{M}^{\perp}$.

Since $(\mathfrak{M})_{n} \perp \mathfrak{N},(\mathfrak{M})_{n} \perp \mathfrak{N}$, we have $(\mathfrak{M})=\sum_{1}^{\infty} *(\mathfrak{M})_{\alpha} \perp \mathfrak{N}$ and $(\mathfrak{M})^{\perp} \supset \mathfrak{N}$. Let $f \varepsilon(\mathfrak{M})^{\perp}$, that is, let $f \varepsilon \mathfrak{B}, f \perp(\mathfrak{M})$. Then $f \perp(\mathfrak{M})_{n}, f_{\varepsilon} \mathfrak{N}_{n}, f \varepsilon \mathfrak{R}$ (see the discussion under II, 2). Hence $(\mathfrak{M})^{\perp}=\mathfrak{R}$.

Since $(\mathfrak{R})=\mathfrak{M}^{\perp},(\mathfrak{R})^{\perp}=\mathfrak{M}^{\perp \perp}=\mathfrak{M}$. It remains to show that $(\mathfrak{R})=\mathfrak{R}^{\perp}$. Starting with $(\mathfrak{M})^{\perp}=\mathfrak{R}$, we obtain $(\mathfrak{M})^{\perp \perp}=\mathfrak{N}^{\perp}$. Since $\mathfrak{B}$ is reflexive, $(\mathfrak{M})^{\perp \perp}=(\mathfrak{M})$ and $(\mathfrak{M})=\mathfrak{N}^{\perp}$.

(3) By the remark preceding this theorem and by (1) and (2), $\mathfrak{M} \mathfrak{+} \mathfrak{N}=\mathfrak{B}$. Similarly, $(\mathfrak{M}) \dot{+}(\mathfrak{R})=(\mathfrak{B})$.

THEOREM 3.2. Let $\left\{P_{n}\right\}$ be a sequence of projections for which $\left|P_{n}\right| \leqq K$, $P_{n}<P_{n+1},(n=1,2, \cdots)$. Then there exists a projection $P$ having the following properties:

(1) $\mathfrak{M}_{P}=\sum_{1}^{\infty} * \mathfrak{M}_{\alpha}, \mathfrak{R}_{P}=\prod_{1}^{\infty} \mathfrak{N}_{\alpha}$.

(2) $|P| \leqq K$.

(3) For any $f \& \mathfrak{B},\left\|\left(P-P_{n}\right) f\right\| \rightarrow 0$.

(4) $P>P_{n},(n=1,2, \cdots)$. If $Q$ is a projection such that $Q>P_{n}$, $(n=1,2, \cdots)$, then $Q>P$.

(5) If $P_{n}$ is permutable with a linear operator $A,(n=1,2, \cdots)$, then $P$ is permutable with $A$.

Similar conclusions may be drawn for sequences $\left\{P_{n}\right\}$ in which the hypothesis $P_{n}>P_{n+1}$ replaces $P_{n}<P_{n+1},(n=1,2, \cdots)$. 
(1) We define $P$ to be the projection whose associated manifolds are $\mathfrak{M}_{P}=\sum_{1}^{\infty} * \mathfrak{M}_{\alpha}, \mathfrak{N}_{P}=\prod_{1}^{\infty} \mathfrak{N}_{\alpha}$. Since these manifolds are disjoint and since $\mathfrak{M}_{P} \dot{+} \mathfrak{N}_{P}=\mathfrak{B}$ (by the previous theorem), $P$ is uniquely defined (Theorem 2.3).

(2) That $|P| \leqq K$ is apparent from the proof of the first statement in Theorem 3.1.

(3) Assume that $f \varepsilon \mathfrak{B}$. Then $P f \varepsilon \mathfrak{M}_{P}$, and there exist elements $g_{n} \varepsilon \mathfrak{M}_{n}$, $(n=1,2, \cdots)$, such that $g_{n} \rightarrow P f$. Now $P f-P_{n} f=P f-g_{n}+P_{n}\left(g_{n}+f-P f\right)-P_{n} f$ and $\left\|P f-P_{n} f\right\| \leqq\left\|P f-g_{n}\right\|+\left\|P_{n}\left(g_{n}-P f\right)\right\| \rightarrow 0$ since $\left|P_{n}\right| \leqq K$.

(4) That $P>P_{n}$ is clear from (1). If $Q>P_{n}$, then $\mathfrak{M}_{Q} \supset \mathfrak{M}_{n},(n=1,2, \cdots)$, hence $\mathfrak{M}_{Q} \supset \mathfrak{M}_{P}$. Similarly, $\mathfrak{N}_{Q} \subset \mathfrak{N}_{P}$. Thus $P<Q$.

(5) Since $P_{n} A f=A P_{n} f, P_{n} A f \rightarrow P A f, A P_{n} f \rightarrow A P f$, then $P A f=A P f$.

The proof of the last statement in the theorem presents no difficulties.

Let $P_{1}, P_{2}, \cdots, P_{n}$ be mutually permutable projections. Then $Q=\prod_{1}^{n} P_{n}$ is a projection. It is readily seen that $\mathfrak{M}_{Q}=\prod_{1}^{n} \mathfrak{M}_{\alpha}, \mathfrak{N}_{Q}=\sum_{1}^{n *} \mathfrak{N}_{\alpha}$. The manifolds associated with the projection $I-P_{1}$ are $\mathfrak{M}_{I-P_{1}}=\mathfrak{N}_{1}, \mathfrak{N}_{I-P_{1}}=\mathfrak{M}_{1}$. Thus the manifolds associated with the projection $R=I-\prod_{1}^{n}\left(I-P_{\alpha}\right)$ are $\mathfrak{M}_{R}=\sum_{1}^{n *} \mathfrak{M}_{\alpha}, \mathfrak{N}_{R}=\prod_{1}^{n} \mathfrak{N}_{\alpha}$. The projection $R$ formed in this way is denoted by the symbol $R=\sum_{1}^{n *} P_{\alpha}$ (or $R=P_{1} \dot{+} \cdots \dot{+} P_{n}$ ).

Definition 3. A set $\Omega$ of permutable projections is called a lattice $\dagger$ of projections if, when $P, P_{1}, P_{2} \varepsilon \Omega$, then $I-P, P_{1} P_{2} \varepsilon \Omega$. The lattice is said to be $K$-bounded if $|P| \leqq K$ for every $P \varepsilon \Omega$.

If $P_{1}, P_{2} \varepsilon \Omega$, then $P_{1} \dot{+} P_{2}=I-\left(I-P_{1}\right)\left(I-P_{2}\right) \varepsilon \Omega$. Any set $\mathfrak{M}$ of permutable projections may be embedded in a lattice of projections. Indeed, let $\mathfrak{B}\left(\xi_{1}, \xi_{2}, \cdots, \xi_{s}\right)$ represent any polynomial with integral coefficients such that $\mathfrak{B}\left(P_{1}, P_{2}, \cdots, P_{s}\right)$ is a projection, for any set of $s$ mutually permutable projections $P_{1}, P_{2}, \cdots, P_{s} . \ddagger$ The set of all projections thus obtained contains $\mathfrak{M}$ and is a lattice of projections.

THEOREM 3.3. Let $\Omega$ be any $K$-bounded lattice of projections, and let $\left\{P_{\alpha}\right\}$ be any subset of $\Omega$. Then the manifolds $\mathfrak{M}=\sum_{\alpha}^{*} \mathfrak{M}_{\alpha}$ and $\mathfrak{N}=\prod_{\alpha} \mathfrak{N}_{\alpha}$ are disjoint and $\mathfrak{M}+\mathfrak{i}=\mathfrak{B}$. Similarly, the manifolds $\mathfrak{M}^{\prime}=\prod_{\alpha} \mathfrak{M}_{\alpha}$ and $\mathfrak{N}^{\prime}=\sum_{\alpha}^{*} \mathfrak{N}_{\alpha}$ are disjoint and $\mathfrak{M}^{\prime}+\mathfrak{N}^{\prime}=\mathfrak{B}$. Let $P$ be the projection associated to $\mathfrak{M}$ and $\mathfrak{R}$; let $P^{\prime}$ be the projection associated to $\mathfrak{M}^{\prime}$ and $\mathfrak{R}^{\prime}$. Then $|P| \leqq K,\left|P^{\prime}\right| \leqq K, P>P_{\alpha}$, and $Q>P_{\alpha}$ implies $Q>P$. Also, $P_{\alpha}>P^{\prime}$, and $P_{\alpha}>Q$ implies $P^{\prime}>Q$. The projections $P$ and $P^{\prime}$ are permutable with any linear operator permutable with $P_{\alpha}$.

Let $f \varepsilon \sum_{\alpha}^{*} \mathfrak{M}_{\alpha}, g \varepsilon \prod_{\alpha} \mathfrak{M}_{\alpha}$. Then there exist elements $f_{n}$ and manifolds $\mathfrak{M}_{r}$, associated to the projections $P_{r} \varepsilon \Omega,(n, r=1,2, \cdots)$, such that $f_{n} \varepsilon \sum_{r=1}^{r_{n}} \mathfrak{M}_{r}$,

$\dagger$ In fact, a lattice of projections constitutes a Boolean algebra.

$\ddagger$ In making the substitutions, write $I$ for $P_{j}{ }^{0}$. 
$f_{n} \rightarrow f$. Let $Q$ be the projection which is the limit of the monotone sequence $\left\{Q_{s}=\sum_{r=1}^{s *} P_{r}\right\}$ (Theorem 3.2). Then $\mathfrak{M}_{Q}=\sum_{r=1}^{\infty *} \mathfrak{M}_{r} \subset \mathfrak{M}$ and $\mathfrak{N}_{Q} \supset \mathfrak{M}$. Since $|Q| \leqq K$ (Theorem 3.2), $\|Q(f+g)\|=\|f\| \leqq K\|f+g\|$. This proves that $\mathfrak{M}$ and $\mathfrak{N}$ are disjoint. Similarly, $\mathfrak{M}^{\prime}$ and $\mathfrak{R}^{\prime}$ are disjoint.

We prove that $\mathfrak{M} \dot{+} \mathfrak{N}=\mathfrak{R}$. Let $(\mathfrak{M})_{\alpha},(\mathfrak{R})_{\alpha}$ be the manifolds associated to $\bar{P}_{\alpha}$. Let $(\mathfrak{R})=\sum_{\alpha}^{*}(\mathfrak{M})_{\alpha},(\mathfrak{R})=\prod_{\alpha}(\mathfrak{M})_{\alpha}$. Then precisely as in the proof of Theorem 3.1, $(\mathfrak{R})=\mathfrak{R}^{\perp},(\mathfrak{R})=\mathfrak{M}^{\perp}$; and since $(\mathfrak{R})$ and $(\mathfrak{R})$ are disjoint (by the argument given above) $\mathfrak{M} \dot{+} \mathfrak{N}=\mathfrak{B}$. Similarly $\mathfrak{M}^{\prime} \dot{+} \mathfrak{R}^{\prime}=\mathfrak{B}$.

That $|P| \leqq K,\left|P^{\prime}\right| \leqq K$ follows from the inequality $\|f\| \leqq K\|f+g\|$ derived in the first paragraph of this proof. That $P>P_{\alpha}, P^{\prime}<P_{\alpha}$ is clear. If $Q>P_{\alpha}$, then $\mathfrak{M}_{Q} \supset \sum_{\alpha}^{*} \mathfrak{M}_{\alpha}=\mathfrak{M}_{P}$ and $\mathfrak{N}_{Q} \subset \prod_{\alpha} \mathfrak{N}_{\alpha}=\mathfrak{N}_{P}$; hence $Q>P$. Similarly if $Q<P_{\alpha}, Q<P^{\prime}$.

We examine the statement on permutability. Let $A P_{\alpha}=P_{\alpha} A, A$ linear, and let $f \varepsilon \mathfrak{B}$. As in the first paragraph of this proof, we obtain a projection $Q_{1}<P$ such that $P f \varepsilon \mathfrak{M}_{Q_{1}}$ or $P f=Q_{1} f$. Similarly, we obtain a $Q_{2}<P$ such that $P A f \varepsilon \mathfrak{M}_{Q_{2}}$ or $P A f=Q_{2} A f$. By Theorem 3.2, $Q_{1}, Q_{2}$, and also $Q=Q_{1} \dot{+} Q_{2}$, are permutable with $A$. We have $Q_{1} f=Q Q_{1} f=Q P f=Q f$ and $Q A f=Q_{2} A f$, and since $Q A=A Q, A P f=A Q_{1} f=A Q f=Q A f=Q_{2} A f=P A f$.

The projections $P$ and $P^{\prime}$ of this theorem will be denoted by the symbols $\sum_{\alpha}^{*} P_{\alpha}$ and $\prod_{\alpha} P_{\alpha}$, respectively.

THEOREM 3.4. Let $\Omega$ be a K-bounded lattice of projections. Let $\Omega^{\prime}$ be the set of all projections of the type $P=\sum_{\alpha}{ }^{*} P_{\alpha}, P^{\prime}=\prod_{\alpha} P_{\alpha}$ where the sums and products are formed over the subsets of $\Omega$. Then $\Omega^{\prime}$ may be embedded in a $K$-bounded lattice of projections.

Letting $P_{t}$ represent an arbitrary projection in $\Omega^{\prime}$, we create independent variables $x_{t}$ in 1-1 correspondence to the $P_{t}$. We define a sequence $\left\{M_{n}\right\}$ of classes of polynomials in the variables $x_{t} ; M_{0}$ is the set of all $x_{t}$; assuming that $M_{n}$ is known, we define $M_{n+1}$. If $n$ is even, $M_{n+1}$ is the set of all polynomials of the form $y_{1}, y_{2}, \cdots, y_{n}$ where $y_{i} \varepsilon M_{n}$. If $n$ is odd, $M_{n+1}$ is the set of all polynomials in $M_{n}$ to which have been added all polynomials of the form $1-y$, where $y_{\varepsilon} M_{n}$. This process defines $M_{n}$ for $n=1,2, \cdots$. Let $M=\sum_{n=1}^{\infty} M_{n}$. Then $M$ has the following properties: $x_{t} \varepsilon M$; if $y, y_{1}, y_{2} \varepsilon M$, then $1-y, y_{1} y_{2} \varepsilon M$. In fact, as one may easily see, $M$ is the smallest set of polynomials possessing these properties. If $y=y\left(x_{t_{1}}, \cdots, x_{t_{m}}\right) \varepsilon M$, then $y\left(P_{t_{1}}, \cdots, P_{t_{m}}\right)$ is a projection. For there exists a value of $n$ such that $y \varepsilon M_{n}$. If $n=0$, cur assertion is obvious. A clearly indicated induction settles the case $n \geqq 1$. Let $\Gamma$ be the set of all projections $y\left(P_{t_{1}}, \cdots, P_{t_{m}}\right)$. We shall show that $\Gamma$ is a $K$-bounded lattice of projections.

Since the members of $\Omega^{\prime}$ may be permuted among themselves, the same is true of the members of $\Gamma$. From the definition of $M$, it is clear that $\Gamma$ is a 
lattice of projections; indeed, it is the smallest lattice which includes $\Omega^{\prime}$. We shall see that for arbitrary $R_{\varepsilon} \Gamma, f_{\varepsilon} \mathfrak{B}$, there exists a $Q \varepsilon \Omega$ such that $\|(R-Q) f\|$ is small at will. Then since $\|R f\| \leqq\|(R-Q) f\|+\|Q f\|,\|R f\| \leqq K\|f\|$.

Let $P=\sum_{\alpha}^{*} P_{\alpha} \varepsilon \Omega^{\prime}, P_{\alpha} \varepsilon \Omega$; let $f \varepsilon \mathscr{B}$. Then as in Theorem 3.3 there exists a monotone increasing sequence $\left\{P_{n}\right\}$ such that $P_{n} \varepsilon \Omega, P_{n} f \rightarrow P f$. If $P^{\prime}=\prod_{\alpha} P_{\alpha} \varepsilon \Omega^{\prime}$, we may find a decreasing sequence $\left\{P_{n}^{\prime}\right\}, P_{n}^{\prime} \varepsilon \Omega, P_{n}^{\prime} f \rightarrow P^{\prime} f$. Now let $R_{1}, \cdots, R_{\mathbf{q}} \varepsilon \Omega^{\prime}, R=y\left(R_{1}, \cdots, R_{\mathbf{s}}\right) \varepsilon \Gamma$. Let $R_{i n} f \rightarrow R_{i} f, R_{i n} \varepsilon \Omega$, $(i=1,2, \cdots, s)$. It is clear from the construction of $\Gamma$ that $y\left(R_{1 n}, \cdots, R_{s n}\right) \varepsilon \Omega$. It may also be seen that $y\left(R_{1 n}, \cdots, R_{e n}\right) f \rightarrow y\left(R_{1}, \cdots, R_{\imath}\right) f$.

\section{THE THEORY OF PROJECTION MEASURE}

In this chapter, we define the notion of a resolution of the identity in $\mathfrak{B}$. A theory of projection measure generated by this resolution of the identity is developed. With certain sets of real numbers we associate projections. Products and sums of sets correspond to products and *-sums of the associated projections. $\dagger$

A set of real numbers $a<\lambda \leqq b$ will be designated by $\delta$. Let $\left\{\delta_{n}\right\}$ be a sequence of such sets; we designate $\sum_{1}^{\infty} \delta_{\alpha}$ by $\Delta$. The set $\Delta$ is said to be a covering of a set $M$ if $\Delta \supset M$.

Definition 4. A set of projections $E(\lambda),(-\infty<\lambda<\infty)$, is called a resolution of the identity if

(1) The projections 0 and $I$ are in the set;

(2) $E(\lambda)>E(\mu)$ for $\lambda>\mu$;

(3) There exists a constant $K$ such that for any given real numbers $a_{i}, b_{i}$, $(i=1,2, \cdots, n)$, with $a_{1} \leqq b_{1} \leqq \cdots \leqq a_{i} \leqq b_{i} \leqq \cdots \leqq a_{n} \leqq b_{n}$, and for any given complex numbers $\mu_{i},(i=1,2, \cdots, n)$, with $\left|\mu_{i}\right| \leqq 1$ the bound of the operator

$$
\sum_{1}^{n} \mu_{\alpha}\left[E\left(b_{\alpha}\right)-E\left(a_{\alpha}\right)\right]
$$

does not exceed $K$.

(1) and (2) imply the existence of two real numbers $r, R$ such that $E(\lambda)=0$ for $\lambda \leqq r, E(\lambda)=I$ for $\lambda \geqq R$.

We shall prove that if $\Delta=\sum_{1}^{\infty} \delta_{\alpha}, \delta_{i}=\left\{a_{i}<\lambda \leqq b_{i}\right\}$ is any covering of the set of all real numbers $\lambda$, and if $E\left(\delta_{i}\right)$ means $E\left(b_{i}\right)-E\left(a_{i}\right)$, then $\sum_{1}^{\infty} * E\left(\delta_{\alpha}\right)=I$. Let us write here, as often later, $E(\Delta)$ for $\sum_{1}^{\infty} * E\left(\delta_{\alpha}\right)$. Then $E\left(\Delta_{n}\right)=\sum_{1}^{n *} E\left(\delta_{\alpha}\right)$ $\rightarrow E(\Delta)$. In the first place, for fixed $f_{\varepsilon} \mathfrak{B}, F \varepsilon(\mathfrak{B})$, the function $F(E(\lambda) f)$ is of bounded variation and indeed $\operatorname{var} F(E(\lambda) f) \leqq K \cdot|F| \cdot\|f\|$. For consider a sub-

$\dagger$ Such a theory was developed by the author for the case in which $\mathbb{B}$ is a (separable) Hilbert space in Acta Litterarum ac Scientiarum, vol. 7 (1935), pp. 136-146. 
division $r=a_{0} \leqq a_{1} \leqq \cdots \leqq a_{n}=R$ of the interval $\beta=\{r \leqq \lambda \leqq R\}$ with $r, R$ as above. Assume

$F\left(E\left(a_{j}\right) f\right)-F\left(E\left(a_{j-1}\right) f\right)=e^{i \theta_{j}}\left|F\left(E\left(a_{j}\right)-E\left(a_{j-1}\right)\right) f\right|, \theta_{j}$ real, $j=1,2, \cdots, n$.

Then

$$
\begin{aligned}
\sum_{j=1}^{n}\left|F\left(E\left(a_{j}\right)-E\left(a_{j-1}\right)\right) f\right| & =\left|\sum_{j=1}^{n} F\left(e^{-i \theta_{j}}\left[E\left(a_{j}\right)-E\left(a_{j-1}\right)\right] f\right)\right| \\
& =|F(T \cdot f)| \leqq|F| \cdot|T| \cdot\|f\|,
\end{aligned}
$$

$T$ being a certain linear transformation defined by the equation. But $|T| \leqq K$ by Definition $4,(3)$. Now writing $\Gamma_{n}=\beta-\sum_{1}^{n} \delta_{\alpha},(n=1,2, \cdots)$, we see that $\Gamma_{1} \supset \Gamma_{2} \supset \cdots \supset \Gamma_{n} \supset \cdots$, and that $\prod_{1}^{\infty} \Gamma_{\alpha}$ is the null set. Hence

$$
\left|F(f)-F E\left(\Delta_{n}\right) f\right|=\left|F\left(I-E\left(\Delta_{n}\right)\right) f\right|=\left|F\left(E\left(\Gamma_{n}\right) f\right)\right| \leqq \underset{\Gamma_{n}}{\operatorname{var}} F(E(\lambda) f) .
$$

Thus $F(f)-F E\left(\Delta_{n}\right) f \rightarrow 0$ with $1 / n$. Since $E\left(\Delta_{n}\right) f$ converges weakly to $f$ and strongly to $E(\Delta) f$, we have $E(\Delta) f=f$, and our proof is complete.

We prove the following theorem:

THEOREM 4.1. Any resolution of the identity may be embedded in a $K$ bounded lattice of projections.

Let $\delta_{1}, \cdots, \delta_{n}$ be nonoverlapping intervals. Then $\sum_{1}^{n *} E\left(\delta_{\alpha}\right)$ is a projection, and its bound does not exceed $K$ (Definition 4 , (3)). The totality $\Omega$ of projections formed in this manner is a $K$-bounded lattice. The members of $\Omega$ are permutable. If $P, P_{1}, P_{2} \varepsilon \Omega$, then $I-P, P_{1} P_{2} \varepsilon \Omega$, since the complement in the set $r<\lambda \leqq R$ of $\sum_{1}^{n} \delta_{\alpha}$ is a set of the same type and since the product of two such sets yields a third.

Let $M$ be any set of real numbers; let $\left\{\Delta_{\alpha}\right\}$ be the set of all coverings of $M$. Then $\prod_{\alpha} E\left(\Delta_{\alpha}\right)$ is a projection by Theorem 3.4. This projection is called the exterior projection measure of $M$, and we write $\prod_{\alpha} E\left(\Delta_{\alpha}\right)=E[M]$. The set of all projections $E[M]$ can be embedded in a $K$-bounded lattice by Theorem 3.4 .

We discuss at this point some matters of future usefulness. If $M_{1} \supset M_{2}$, then $E\left[M_{1}\right]>E\left[M_{2}\right]$. It is also clear that for any interval $\delta, E[\delta]<E(\delta)$. We shall show that $E[\delta]=E(\delta)$. Let $\Delta=\sum_{1}^{\infty} \delta_{\alpha}$ be any covering of $\delta=\{a<\lambda \leqq b\}$. We may and shall assume that $\Delta=\delta$. Let $\delta_{0}=\{r<\lambda \leqq a\}, \delta_{-1}=\{b<\lambda \leqq R\}$. Then by the discussion preceding this theorem,

Since

$$
\sum_{-1}^{\infty} E\left(\delta_{\alpha}\right)=E\left(\delta_{-1}\right) \dot{+} E\left(\delta_{0}\right)+\sum_{1}^{\infty} E\left(\delta_{\alpha}\right)=I \text {. }
$$




$$
\begin{aligned}
E\left(\delta_{-1}\right)+E\left(\delta_{0}\right)+E(\delta)=I, \quad E\left(\delta_{i}\right) E(\delta)=E\left(\delta_{i}\right) E\left(\delta_{j}\right) & =0, \\
i & =-1,0 ; j=1,2, \cdots,
\end{aligned}
$$

we conclude $\sum_{1}^{\infty} * E\left(\delta_{\alpha}\right)=E(\delta)$. Hence $E[\delta]=E(\delta)$.

Similarly, if $\Delta=\sum_{1}^{\infty} \delta_{\alpha}$, then $E[\Delta]=E(\Delta)\left(=\sum_{1}^{\infty} * E\left(\delta_{\alpha}\right)\right.$ by definition). Clearly $E[\Delta]<E(\Delta)$. Let $\sum_{1}^{\infty} \delta_{\alpha}^{\prime}$ be a covering of $\Delta$; then $\sum_{1}^{\infty} \delta_{\alpha}^{\prime} \supset \delta_{i}$, $(i=1,2, \cdots)$; hence $\sum_{1}^{\infty} * E\left(\delta_{\alpha}^{\prime}\right)>E\left[\delta_{i}\right]=E\left(\delta_{i}\right)$. Thus $\sum_{1}^{\infty} * E\left(\delta_{\alpha}^{\prime}\right)>\sum_{1}^{\infty} * E\left[\delta_{\alpha}\right]$ $=\sum_{1}^{\infty} * E\left(\delta_{\alpha}\right)=E(\Delta)$.

For any set $M$, and any element $f \varepsilon \mathfrak{B}$, there exists a sequence of coverings $\left\{\Delta_{n}^{\prime}\right\}$ of $M$ such that $E\left(\Delta_{n}^{\prime}\right) f \rightarrow E[M] f$. For there exist coverings $\Delta_{n}$ such that $\prod_{1}^{n} E\left(\Delta_{\alpha}\right) f \rightarrow E[M] f$ (see proof of Theorem 3.3). Now $\prod_{1}^{n} \Delta_{\alpha}$ is a set of the " $\Delta$ type"; write $\Delta_{n}^{\prime}=\prod_{i}^{n} \Delta_{\alpha}$. Then we obtain $\prod_{1}^{n} E\left(\Delta_{\alpha}\right)>E\left[\Delta_{n}^{\prime}\right]=E\left(\Delta_{n}^{\prime}\right)>$ $E[M]$. Hence $E\left(\Delta_{n}^{\prime}\right) f \rightarrow E[M] f$.

If $A_{n}, B_{n}$ are projections in a $K$-bounded lattice $\Omega$ such that for a given $f \varepsilon \mathfrak{B},\left\|\left(A_{n}-B_{n}\right) f\right\| \leqq \epsilon_{n},(n=1,2, \cdots)$, then for any $C \varepsilon \Omega$,

$$
\left\|C\left(\sum_{1}^{n} A_{\alpha}-\sum_{1}^{n} * B_{\alpha}\right) f\right\| \leqq K\left(\epsilon_{1}+2 \epsilon_{2}+\cdots+2^{n-1} \epsilon_{n}\right) .
$$

For $n=1,\left\|\left(A_{1}-B_{1}\right) f\right\| \leqq \epsilon_{1},\left\|C\left(A_{1}-B_{1}\right) f\right\| \leqq K \epsilon_{1}$. Assume the statement for $n-1$. Then

$$
\begin{aligned}
C\left(\sum_{1}^{n} *_{\alpha}-\sum_{1}^{n} B_{\alpha}\right) f=C & \left(\sum_{2}^{n} A_{\alpha}-\sum_{2}^{n} * B_{\alpha}\right) f+C\left(A_{1}-B_{1}\right) f \\
& -C\left(A_{1} \sum_{2}^{n} A_{\alpha}-B_{1} \sum_{2}^{n} B_{\alpha}\right) f .
\end{aligned}
$$

Hence

$$
\begin{aligned}
\left\|C\left(\sum_{1}^{n} A_{\alpha}-\sum_{1}^{n} B_{\alpha}\right) f\right\| & \leqq K\left(\epsilon_{2}+\cdots+2^{n-2} \epsilon_{n}\right) \\
& +\left\|\left[C A_{1}\left(I-\sum_{2}^{n} A_{\alpha}\right)-C B_{1}\left(I-\sum_{2}^{n} B_{\alpha}\right)\right] f\right\| .
\end{aligned}
$$

The last term above does not exceed $K \epsilon_{1}+K\left(\epsilon_{2}+\cdots+2^{n-2} \epsilon_{n}\right)$.

TheOREM 4.2. For any set $M$ and its complement $\bar{M}, E[M] \dot{+} E[\bar{M}]=I$.

For any $f \varepsilon \mathscr{B}$, we choose sequences $\left\{\Delta_{n}\right\}, \Delta_{n} \supset M,\left\{\Delta_{n}^{\prime}\right\}, \Delta_{n}^{\prime} \supset \bar{M}$, such that $E\left(\Delta_{n}\right) f \rightarrow E[M] f, E\left(\Delta_{n}^{\prime}\right) f \rightarrow E[\bar{M}] f$. By the discussion preceding Theorem 4.1, $E\left(\Delta_{n}\right) f \dot{+} E\left(\Delta_{n}^{\prime}\right) f=f$. But

$$
E\left(\Delta_{n}\right) f \dot{+} E\left(\Delta_{n}^{\prime}\right) f \rightarrow E[M] f \dot{+} E[\bar{M}] f
$$

by the last remark preceding this theorem. This establishes our assertion. 
THEOREM 4.3. If $M=\sum_{1}^{\infty} M_{\alpha}, E[M]=\sum_{1}^{\infty} * E\left[M_{\alpha}\right]$.

Since $M \supset M_{n}, E[M]>E\left[M_{n}\right],(n=1,2, \cdots)$; hence $E[M]>\sum_{1}^{\infty} * E\left[M_{\alpha}\right]$. For fixed $f \& \bar{M}_{E[M]}$ we find coverings $\Delta_{n} \supset M_{n},(n=1,2, \cdots)$, such that $\left\|\left(E\left(\Delta_{n}\right)-E\left[M_{n}\right]\right) f\right\| \leqq \epsilon_{n}$ where $\epsilon_{n} \geqq 0$. Since $\sum_{1}^{\infty} \Delta_{\alpha} \supset M, \sum_{1}^{n *} E\left(\Delta_{\alpha}\right) f=f$. Thus for $\epsilon>0$ we may find an integer $n$ such that $\left\|f-\sum_{1}^{n *} E\left(\Delta_{\alpha}\right) f\right\| \leqq \epsilon$. Finally,

$$
\begin{aligned}
\left\|f-\sum_{1}^{n} * E\left[M_{\alpha}\right] f\right\| & \leqq\left\|f-\sum_{1}^{n} * E\left(\Delta_{\alpha}\right) f\right\|+\left\|\sum_{1}^{n} * E\left(\Delta_{\alpha}\right) f-\sum_{1}^{n} * E\left[M_{\alpha}\right] f\right\| \\
& \leqq \epsilon+K\left(\epsilon_{1}+2 \epsilon_{2}+\cdots+2^{n-1} \epsilon_{n}\right) \leqq \epsilon(1+K)
\end{aligned}
$$

if $\epsilon_{i}<\epsilon / 2^{2 n-1}$. Thus $\sum_{1}^{\infty} * E\left[M_{\alpha}\right] f=f$, and $E[M]=\sum_{1}^{\infty} * E\left[M_{\alpha}\right]$.

A set $M$ is said to be projection measurable if $E[M] \cdot E[\bar{M}]=0$ where $\bar{M}$ denotes the complement of $M$. If $M$ is projection measurable, its projection measure is defined to be $E[M]$. If $M$ is projection measurable, $\bar{M}$ is projection measurable. Since $E[M] \dot{+} E[\bar{M}]=I, E[M] \cdot E[\bar{M}]=0, E[\bar{M}]=I-E[M]$. Any set $\delta$ is projection measurable.

THEOREM 4.4. If $M_{n}$ is projection measurable, $(n=1,2, \cdots)$, then $M=\sum_{1}^{\infty} M_{\alpha}$ is projection measurable and $E[M]=\sum_{1}^{\infty} *\left[M_{\alpha}\right]$. In addition $M^{\prime}=\prod_{1}^{\infty} M_{\alpha}$ is projection measurable and $E\left[M^{\prime}\right]=\prod_{1}^{\infty} E\left[M_{\alpha}\right]$.

We have $\bar{M}=\prod_{1}^{\infty} \bar{M}_{\alpha}$, hence $E[\bar{M}]<\prod_{1}^{\infty} E\left[\bar{M}_{\alpha}\right]$. By Theorem 4.3, $E[M]$ $=\sum_{1}^{\infty} E\left[M_{\alpha}\right]$. We shall prove that $E[M] \cdot \prod_{1}^{\infty} E\left[\bar{M}_{\alpha}\right]=0$. This will imply $E[M] \cdot E[\bar{M}]=0, M$ projection measurable. For fixed $\epsilon>0, f \varepsilon \mathfrak{B}$, we determine $n$ so that

Then

$$
\left\|E[M] f-\sum_{1}^{n} * E\left[M_{\alpha}\right] f\right\| \leqq \epsilon .
$$

$$
\begin{aligned}
\left\|E[M] \cdot \prod_{1}^{\infty} E\left[\bar{M}_{\alpha}\right] f\right\| \leqq & \left(E[M]-\sum_{1}^{n} E\left[M_{\alpha}\right]\right) \prod_{1}^{\infty} E\left[\bar{M}_{\alpha}\right] f \| \\
& +\left\|\left(\sum_{1}^{n} * E M_{\alpha}\right) \cdot \prod_{1}^{\infty} E\left[\bar{M}_{\alpha}\right] f\right\| \leqq K \epsilon .
\end{aligned}
$$

For $M^{\prime}$, we have $\bar{M}^{\prime}=\sum_{1}^{\infty} \bar{M}_{\alpha}$, and $\bar{M}^{\prime}$ is projection measurable according to what precedes; hence $M^{\prime}$ is projection measurable, and

$$
E\left[\bar{M}^{\prime}\right]=\sum_{1}^{\infty} E\left[\bar{M}_{\alpha}\right]=\sum_{1}^{\infty}\left(I-E\left[M_{\alpha}\right]\right) .
$$

Thus if the manifolds associated to $E\left[M_{n}\right]$ are $\mathfrak{M}_{n}, \mathfrak{N}_{n}$, those associated to $\sum_{1}^{\infty} *\left(I-E\left[M_{\alpha}\right]\right)$ are $\sum_{1}^{\infty} * \mathfrak{N}_{\alpha}, \prod_{1}^{\infty} \mathfrak{M}_{\alpha}$, and those associated to $E\left[M^{\prime}\right]=I$ $-E\left[\bar{M}^{\prime}\right]$ are $\prod_{1}^{\infty} \mathfrak{M}_{\alpha}, \sum_{1}^{\infty} * \mathfrak{R}_{\alpha}$. In other words, $E\left[M^{\prime}\right]=\prod_{1}^{\infty} E\left[M_{\alpha}\right]$. 
From this point forward, and for obvious reasons, we shall denote the projection measure of a projection measurable set $M$ by $E(M)$. Thus the new symbol $E(M)$ is identical with the old whenever both are significant. We note that Borel sets are projection measurable. We shall use the terms "of zero measure," "almost everywhere," and so on, without introducing them formally.

\section{The OPERATIONAL CALCULUS}

In this chapter we establish the existence of an extensive homomorphism between a substantial class of real functions and a class of operators. As our principal theorem indicates, the correspondence is more than a ring homomorphism.

To a resolution of the identity $E(\lambda)$, we associate an operator $A$ in the following manner: Let $\delta_{1}, \cdots, \delta_{n}$ be nonoverlapping intervals covering the fundamental interval $r<\lambda \leqq R$ (where $E(r)=0, E(R)=I)$. Let $\lambda_{i} \varepsilon \delta_{i}$, $(i=1, \cdots, n)$, and form the operator $\sum_{1}^{n} \lambda_{\alpha} E\left(\delta_{\alpha}\right)$. Consider, as in the classic case, a sequence of such divisions of the fundamental interval in which the maximum length of any interval converges to zero. The sequence of operators which corresponds to the sequence of subdivisions converges by virtue of Definition 4, (3) to a linear operator $A$ whose bound does not exceed $K \cdot \max (|r|,|R|)$. Because of its suggestive value, we may, if we wish, write $A=\int \lambda d E(\lambda)$.

We now apply our theory of measure to real functions $\phi(\lambda)$ of the real variable $\lambda$. The function $\phi(\lambda)$ is said to be $E(\lambda)$-measurable if the sets $M_{\mu}=\{\phi(\lambda) \leqq \mu\}$ are projection measurable. We consider exclusively functions $\phi(\lambda)$ which are measurable and bounded almost everywhere. If $\phi_{1}(\lambda)$ and $\phi_{2}(\lambda)$ are two such functions, so are $\phi_{1}(\lambda)+\phi_{2}(\lambda), \phi_{1}(\lambda) \cdot \phi_{2}(\lambda)$. Any Baire function is $E(\lambda)$-measurable. The limit of a converging sequence of $E(\lambda)$-measurable functions is $E(\lambda)$-measurable.

THEOREM 5.1. Let $\phi(\lambda)$ be $E(\lambda)$-measurable and bounded almost everywhere. Let $M_{\mu}=\{\phi(\lambda) \leqq \mu\},(-\infty<\mu<\infty)$. Then $D(\mu)=E\left(M_{\mu}\right)$ is a resolution of the identity.

Since $\phi(\lambda)$ is bounded, $|\phi(\lambda)|<s$ almost everywhere. To establish (1) in Definition 4, note that $D(-s)=E\left(M_{-s}\right)=0, D(s)=E\left(M_{s}\right)=I$. (2) Since $M_{\mu} \subset M_{\nu}$ for $\mu<\nu$, we have $D(\mu)<D(\nu)$. (3) Let $\delta_{1}, \cdots, \delta_{n}$ be nonoverlapping intervals in the $\mu$-space and $\mu_{1}, \cdots, \mu_{n}$ complex numbers for which $\left|\mu_{i}\right| \leqq 1$. Then to the $\delta_{i}$ correspond measurable sets $M_{i}$ in the $\lambda$-space for which $M_{i} M_{j}=0,(i \neq j)$. For fixed $f \varepsilon B$ and $\epsilon>0$ we find $\Delta_{i}=\sum_{1}^{\infty} \delta_{i \alpha},(i=1, \cdots, n)$, such that 


$$
\left\|\left[\sum_{1}^{\infty} E\left(\delta_{i \alpha}\right)-D\left(\delta_{i}\right)\right] f\right\|<\epsilon
$$

(we assume that $\delta_{i j} \delta_{i k}=0,(j \neq k)$ ). We then find suitable integers $n_{i}$ such that

$$
\left\|\left[\sum_{1}^{n_{i}} E\left(\delta_{i \alpha}\right)-D\left(\delta_{i}\right)\right] f\right\|<\epsilon .
$$

Let us replace $\sum_{1}^{n *} E\left(\delta_{i \alpha}\right)$ by $E_{i}, D\left(\delta_{i}\right)$ by $D_{i}$. Then

$$
\begin{aligned}
\sum_{1}^{n} \mu_{\alpha} D_{\alpha}= & \sum_{1}^{n} \mu_{\alpha}\left(D_{\alpha}-E_{\alpha}\right) \\
& +\left[\mu_{1} E_{1}+\mu_{2}\left(E_{2}-E_{1} E_{2}\right)+\cdots+\mu_{n}\left(E_{n}-E_{n} \sum_{1}^{n-1} E_{\alpha}\right)\right] \\
& +\mu_{2} E_{2} E_{1}+\cdots+\mu_{n} E_{n} \sum_{1}^{n-1} E_{\alpha} .
\end{aligned}
$$

From the terms on the right side of the equation we obtain $\left\|\sum_{1}^{n} \mu_{\alpha}\left(D_{\alpha}-E_{\alpha}\right) f\right\|$ $\leqq n \epsilon$. The operator in brackets yields, when applied to $f$, an element of norm at most $K\|f\|$ by Definition 4, (3). In examining the norm of the element $E_{i} \sum_{1}^{i-1 *} E_{\alpha} f,(i=2, \cdots, n)$, we use the fact that $E\left(M_{i}\right) \sum_{1}^{i-1 *} E\left(M_{\alpha}\right)=0$ as well as an inequality immediately preceding Theorem 4.2 . We have

$$
\begin{aligned}
\left\|E_{i}\left(\sum_{1}^{i-1} E_{\alpha}\right) f\right\| & \leqq \\
& \quad+\| E_{i}\left(\sum_{1}^{i-1} \sum_{1}^{*} E\left(M_{\alpha}\right)\left(E_{i}-E\left(M_{i}\right)\right) f \|\right. \\
& \leqq K \epsilon\left(1+2+\cdots+2^{i-2}\right)+K \epsilon \\
& =2^{i-1} K \epsilon .
\end{aligned}
$$

Thus $\left\|\left(\sum_{1}^{n} \mu_{\alpha} D_{\alpha}\right) f\right\| \leqq n \epsilon+K\|f\|=2\left(2^{n-1}-1\right) K \epsilon$. This proves (3) since $n$ is fixed at the outset.

As $E(\lambda)$ yields an operator $A, A=\int \lambda d E(\lambda)$, so does $D(\mu)$ generate an operator which we designate by $\phi(A), \phi(A)=\int \mu d D(\mu)$. We have thus established a correspondence between $E(\lambda)$-measurable functions $\phi(\lambda)$ and operators $\phi(A)$. We write this correspondence in the form $\phi(\lambda) \sim \phi(A)$. In particular, we have $\lambda \sim A$. In what follows, the statements $\phi(\lambda)=\psi(\lambda), \phi(\lambda)<\psi(\lambda)$, $\phi_{n}(\lambda) \rightarrow \phi(\lambda), \cdots$ will imply equality, inequality, convergence, and so on, respectively, almost everywhere.

THEOREM 5.2. Let $E(\lambda)$ be a resolution of the identity, and let $A=\int \lambda d E(\lambda)$. Let $\phi(\lambda), \psi(\lambda), \phi_{n}(\lambda)^{*}$ be any bounded $E(\lambda)$-measurable functions. The corre- 
spondence $\sim$ which associates to these functions the operators $\phi(A), \psi(A), \phi_{n}(A)$ has the following properties:

(1) If $|\phi(\lambda)| \leqq s$, then $|\phi(A)| \leqq K s$.

(2) $\phi(\lambda)+\psi(\lambda) \sim \phi(A)+\psi(A)$.

(3) $\phi(\lambda) \cdot \psi(\lambda) \sim \phi(A) \cdot \psi(A)$.

(4) If $\left|\phi_{n}(\lambda)\right|<b$ and if $\left\{\phi_{n}(\lambda)\right\}$ converges to $\phi(\lambda)$, then $\left\{\phi_{n}(A)\right\}$ converges to $\phi(A)$.

(5) $\phi(A)$ is permutable with any linear operator with which $A$ is permutable.

(6) If $D(\mu)$ is the resolution of the identity of $B=\phi(A)$ and if $\xi(\mu)$ is $D(\mu)$ measurable, then $\xi(\phi(\lambda))$ is $E(\lambda)$-measurable and $\xi(\phi(\lambda)) \sim \xi(B)$.

(7) $\phi(A)=0$ if and only if $\phi(\lambda)=0$.

(8) $\phi(A)$ is a projection if and only if $\phi(\lambda)$ assumes only the values 0 and 1 .

If $\phi(\lambda)$ is a function assuming the values $\lambda_{1}, \cdots, \lambda_{n}$ only on the sets $M_{1}, \cdots, M_{n}$, respectively, then $\phi(A)=\sum_{1}^{n} \lambda_{\alpha} E\left(M_{\alpha}\right)$. If $\phi(\lambda)$ is arbitrary, we may approximate uniformly to $\phi(\lambda)$ by functions $\phi_{n}(\lambda)$ assuming only a finite number of values. $\phi_{n}(\lambda)$ may, for instance, be defined as follows: On $\{q / n<\phi(\lambda) \leqq(q+1) / n\},(q=0, \pm 1, \pm 2, \cdots), \phi_{n}(\lambda)=q / n$ for $q=0,1,2, \cdots$, and $\phi_{n}(\lambda)=(q+1) / n$ for $q=-1,-2, \ldots$. By the definition of $\phi(A)$, $\phi_{n}(A) \rightarrow \phi(A)$. We note that if $|\phi(\lambda)| \leqq s,\left|\phi_{n}(\lambda)\right| \leqq s$, by Theorem 5.1, $\left|\phi_{n}(A)\right| \leqq K s,(n=1,2, \cdots)$; hence $|\phi(A)| \leqq K s$. This proves (1).

(2) Given $\phi(\lambda)$ and $\psi(\lambda)$, let $\left\{\phi_{n}(\lambda)\right\}$ and $\left\{\psi_{n}(\lambda)\right\}$ be chosen as indicated in the previous paragraph. Then $\phi_{n}(\lambda)+\psi_{n}(\lambda) \rightarrow \phi(\lambda)+\psi(\lambda)$ uniformly. The functions $\phi_{n}(\lambda)+\psi_{n}(\lambda),(n=1,2, \cdots)$, assume only a finite number of values, and clearly $\phi_{n}(\lambda)+\psi_{n}(\lambda) \sim \phi_{n}(A)+\psi_{n}(A)=B_{n}$. If we write $\phi(\lambda)+\psi(\lambda) \sim C$, then by the first paragraph, $C$ is the limit of a sequence of operators $C_{n}$ which has the property that $\left|B_{n}-C_{n}\right| \rightarrow 0$. Hence $B_{n} \rightarrow C$ or $C=\phi(A)+\psi(A)$.

(3) The relation is derived by replacing, in the previous paragraph, $\phi+\psi$ by $\phi \cdot \psi, \phi_{n}+\psi_{n}$ by $\phi_{n} \cdot \psi_{n}$.

(4) First, let $\left\{\phi_{n}(\lambda)\right\}$ be a monotone decreasing sequence of positive functions, $b \geqq \phi_{1}(\lambda) \geqq \phi_{2}(\lambda) \geqq \cdots$, for which $\phi_{n}(\lambda) \rightarrow 0$. Let $f \varepsilon \mathfrak{B}$ and $\epsilon>0$. Let $M_{n}=\left\{\phi_{n}(\lambda)>\epsilon\right\}$. Then $M_{1} \supset M_{2} \supset \cdots$, and, since $\phi_{n}(\lambda) \rightarrow 0, \prod_{1}^{\infty} M_{\alpha}=0$; hence $\prod_{1}^{\infty} E\left(M_{\alpha}\right)=0$. Thus there exists an integer $r$ such that

Now

$$
\left\|\prod_{1}^{r} E\left(M_{\alpha}\right) f\right\|=\left\|E\left(M_{r}\right) f\right\| \leqq \epsilon .
$$

$$
\begin{aligned}
\left\|\phi_{r+\bullet}(A) f\right\| & \leqq\left\|\phi_{r+s}(A)\left(I-E\left(M_{r}\right)\right) f\right\|+\left\|\phi_{r+\bullet}(A) E\left(M_{r}\right) f\right\| \\
& \leqq \epsilon K(\|f\|+b), \quad s=1,2, \cdots,
\end{aligned}
$$

by applying to the first term on the right of the inequality the results (3) 
and (1). Thus $\phi_{n}(A) \rightarrow 0$. Similarly if $-b \leqq \phi_{1}(\lambda) \leqq \phi_{2}(\lambda) \leqq \cdots$ and $\phi_{n}(\lambda) \rightarrow 0$, then $\phi_{n}(A) \rightarrow 0$.

Now let $\left\{\phi_{n}(\lambda)\right\},\left|\phi_{n}(\lambda)\right| \leqq b$, be arbitrary subject to the restriction $\phi_{n}(\lambda) \rightarrow 0$. Let $\bar{\psi}_{n}(\lambda)=\max \left\{\phi_{n}(\lambda), \phi_{n+1}(\lambda), \cdots\right\}, \underline{\psi}_{n}(\lambda)=\min \left\{\phi_{n}(\lambda), \phi_{n+1}(\lambda), \cdots\right\}$. Then $\underline{\psi}_{n}(\lambda) \leqq \phi_{n}(\lambda) \leqq \bar{\psi}_{n}(\lambda), \bar{\psi}_{n}(\lambda) \rightarrow 0, \quad \underline{\psi}_{n}(\lambda) \rightarrow 0, \bar{\psi}_{1}(\lambda) \geqq \bar{\psi}_{2}(\lambda) \geqq \cdots$, and $\underline{\psi}_{1}(\lambda) \leqq \underline{\psi}_{2}(\lambda) \leqq \cdots$. Thus for fixed $f \varepsilon \mathfrak{B}$ and $\epsilon>0$, we may find an integer $r$ and sets $\bar{M}_{r}, \underline{M}_{r}$ such that $\left\|E\left(\bar{M}_{r}\right) f\right\| \leqq \epsilon,\left\|E\left(\underline{M}_{r}\right) f\right\|<\epsilon, \bar{\psi}_{r}(\lambda) \leqq \epsilon$ except on $\bar{M}_{r}, \underline{\psi}_{r}(\lambda) \geqq-\epsilon$ except on $\underline{M}_{r}$. Then

$$
\begin{aligned}
&\left\|\phi_{r+8}(A) f\right\| \leqq\left\|\phi_{r+8}(A)\left(I-\left[E\left(\bar{M}_{r}\right)+E\left(\underline{M}_{r}\right)\right]\right) f\right\|+\left\|\phi_{\phi_{r+s}}(A)\left[E\left(\bar{M}_{r}\right)+E\left(\underline{M}_{r}\right)\right] f\right\| \\
& \leqq \epsilon K(\|f\|+2 b), \\
& s=1,2, \cdots .
\end{aligned}
$$

Thus again $\phi_{n}(A) \rightarrow 0$.

In the general case, $\phi_{n}(\lambda) \rightarrow \phi(\lambda)$; hence $\psi_{n}(\lambda)=\phi_{n}(\lambda)-\phi(\lambda) \rightarrow 0$. By (2) and above, $\psi_{n}(A)=\phi_{n}(A)-\phi(A) \rightarrow 0, \phi_{n}(A) \rightarrow \phi(A)$. This completes the proof of statement (4).

(5) In the first place, a linear operator $B$ is permutable with $A, B A=A B$, if and only if $B E(\lambda)=E(\lambda) B,(-\infty<\lambda<\infty)$. If the latter equation holds, so does the former from the very definition of $A$. We prove the converse. For any $\mu$, let $\left\{\phi_{\mu n}(\lambda)\right\}$ be a sequence of polynomials such that $\left|\phi_{\mu n}(\lambda)\right| \leqq C$ in the interval $r<\lambda \leqq R$, and $\phi_{\mu n}(\lambda) \rightarrow \phi_{\mu}(\lambda)$ where $\phi_{\mu}(\lambda)=1,(\lambda \leqq \mu), \phi_{\mu}(\lambda)=0$, $(\lambda>\mu)$. We have $\phi_{\mu}(A)=E(\mu), \phi_{\mu n}(A) \rightarrow E(\mu)$ by $(4)$, and $\phi_{\mu n}(A) B=B \phi_{\mu n}(A)$ by (2) and (3). Hence $E(\mu) B=B E(\mu)$.

If $D(\mu)$ is the resolution of the identity of $\phi(A)$, then $D(\mu)$, and hence $\phi(A)$, are permutable with any linear operator permutable with $E(\lambda)$ (Theorem 3.3 and others), and hence, by the above, permutable with any linear operator permutable with $A$.

(6) Let $\phi(\lambda)$ be $E(\lambda)$-measurable, and let $D(\mu)$ be the resolution of the identity of $\phi(A)$. Let $M$ be any $D(\mu)$-measurable set, and let $N$ denote the set of all numbers $\lambda$ such that $\phi(\lambda) \in M$. We shall show that $N$ is $E(\lambda)$-measurable and that $D(M)=E(N)$. As before, we denote the exterior measure of a set $H$ by $D[H]$ (or $E[H]$ ); use of the symbol $D(H)$ (or $E(H)$ ) will imply that $H$ is measurable. Assume $f_{\varepsilon} \mathfrak{B}$ and $\epsilon>0$. Then there exists a set $\Delta=\Delta(\epsilon)$ covering $M$ such that $\|D(\Delta) f-D(M) f\|<\epsilon$. Let $\Gamma=\Gamma(\epsilon)$ denote the set of all $\lambda$ such that $\phi(\lambda) \varepsilon \Delta$. Then $\Gamma$ is $E(\lambda)$-measurable, $D(\Delta)=E(\Gamma)$, and $\Gamma \supset N$. Let $\left\{\epsilon_{n}\right\}$ be a sequence converging to 0 with $1 / n$, and let $\Theta_{f}=\prod_{1}^{\infty} \Delta\left(\epsilon_{\alpha}\right), \Psi_{f}=\prod_{1}^{\infty} \Gamma\left(\epsilon_{\alpha}\right)$. Then $\Theta_{f} \supset M, \Psi_{f} \supset N, D\left(\Theta_{f}\right) f=D(M) f=E\left(\Psi_{f}\right) f$. Let $\Theta=\prod_{f} \Theta_{f}, \Psi=\prod_{f} \Psi_{f}$, where products are taken over the entire space 2 . Then $\Theta_{f} \supset \Theta \supset M$, $\Psi_{f} \supset \Psi \supset N$. Therefore $\prod_{f} D\left(\Theta_{f}\right)>D[\Theta]>D(M)$. But we see readily that $\prod_{f} D\left(\Theta_{f}\right)=D(M)$. Hence $D(M)=\prod_{f} D\left(\Theta_{f}\right)=\prod_{f} E\left(\Psi_{f}\right)>E[\Psi]>E[N]$. 
If we now turn our attention to $\bar{M}$, the set of all $\lambda$ such that $\phi(\lambda) \varepsilon \bar{M}$ is precisely $\bar{N}$. The argument just given leads to the conclusion $D(\bar{M})>E[\bar{N}]$. Since $M$ is measurable, $D(M) \cdot D(\bar{M})=0$; hence $N$ is measurable and $E(N)$ $=D(M)$.

If $\xi(\mu)$ is $D(\mu)$-measurable, the set $M_{\nu}=\{\xi(\mu) \leqq \nu\}$ is $D(\mu)$-measurable, the set $N_{\nu}=\{\xi(\phi(\lambda)) \leqq \nu\}$ is $E(\lambda)$-measurable and $E\left(N_{\nu}\right)=D\left(M_{\nu}\right)$. Thus $\xi(\phi(\lambda))$ is $E(\lambda)$-measurable. The operator $\xi(B)$, where $B=\phi(A)$, has the resolution of the identity $J(\nu)=D\left(M_{\nu}\right)$. The operator corresponding to the function $\xi(\phi(\lambda))$ has the resolution of the identity $J^{\prime}(\nu)=E\left(N_{\nu}\right)$. Since $J(\nu)=J^{\prime}(\nu)$, the operators are identical or $\xi(\phi(\lambda)) \sim \xi(B)$.

(7) Suppose $\phi(\lambda)=0$; then clearly $\phi(A)=0$. Now assume that $\phi(\lambda) \neq 0$; in this case we may assume that $M=\{|\phi(\lambda)| \geqq 1\}$ has an $E(\lambda)$ measure different from zero, for any other case quickly reduces to this one. Let $\mathfrak{B}(\lambda)$ be the function defined by $\mathfrak{B}(\lambda)=0$ for $|\lambda|<1$ and $\mathfrak{B}(\lambda)=1$ for $\lambda \geqq 1$, and let $\mathfrak{B}_{n}(\lambda)$ be polynomials such that $\mathfrak{B}_{n}(\lambda) \rightarrow \mathfrak{B}(\lambda),\left|\mathfrak{B}_{n}(\lambda)\right| \leqq C$ on the interval $r<\lambda \leqq R$, and $\mathfrak{P}_{n}(0)=0$. Then $\mathfrak{P}_{n}(\phi(\lambda)) \rightarrow \mathfrak{B}(\phi(\lambda))$, and if we write $B=\phi(A)$, we deduce, using (6) and (4), that $\mathfrak{B}_{n}(B)=\mathfrak{B}_{n}(\phi(A)) \rightarrow E(M) \neq 0$. If $B=0, \mathfrak{B}_{n}(B)$ $=0$ by (2) and (3); hence $B=\phi(A) \neq 0$.

(8) If $\phi(\lambda)$ assumes the values 0 and 1 only, then if $\phi(\lambda) \sim R$, since $(\phi(\lambda))^{2}=\phi(\lambda), R^{2}=R$ by (3) and $R$ is a projection. If $R \sim \phi(\lambda)$ and $R$ is a projection, then by (2) and (3), $0=R^{2}-R \sim(\phi(\lambda))^{2}-\phi(\lambda)=\psi(\lambda)$. By (7) $\psi(\lambda)=0$; hence $\phi(\lambda)$ assumes the values 0 and 1 only.

As the aim of this presentation has been to establish the possibility of developing an operational calculus in reflexive spaces, we have purposely refrained from doing this in its most general form. Obvious generalizations of our results will present themselves to the reader; these offer, for the most part, no difficulties.

Barnard College, Columbia University, NEW YoRK, N. Y. 